\title{
ALFA detector before LHC Run 2
}

\section{Vit Vorobel ${ }^{1}$ on behalf of ATLAS collaboration}

Faculty of Mathematics and Physics, Charles University, Prague

12116 Praha 2, Ke Karlovu 3, Czech Republic

E-mail: vit.vorobelemff.cuni.cz

The operation experience with ATLAS ALFA detectors in the LHC environment during the Run 1 period has shown significant beam-induced heating. Subsequent comprehensive studies revealed that heating effects could be disastrous in the case of the larger beam intensities foreseen for higher luminosities in the LHC Run 2. During the first LHC long shutdown (LS1) all ALFA detectors were removed from the LHC tunnel and their covers - Roman Pots - underwent a geometry upgrade to minimize the impedance losses. It is shown that this modification, together with an addition of a system improving the internal heat transfer and an air cooling system, has significantly shifted the temperatures of ALFA detectors away from the critical limits throughout the LHC Run 2. Also ALFA trigger system has been considerably upgraded to keep trigger signals safely inside the Run 2 ATLAS latency budget and to minimize dead time. The hardware changes of the trigger system are also described.

38th International Conference on High Energy Physics

3-10 August 2016

Chicago, USA

\section{${ }^{1}$ Speaker}




\section{ALFA detector system}

The ALFA detector system (Absolute Luminosity For ATLAS [1]) is part of the ATLAS experiment at the LHC [2]. The ALFA is a tracking detector designed to register tracks of protons that are scattered in the very forward direction - scattering angle of order mrad. This allows investigation of elastic scattering in the region of Coulomb-Nuclear interference and to some extent also diffraction processes. The results of the Run 1 data taking at $7 \mathrm{TeV}$ and $8 \mathrm{TeV}$ are published [3, 4].

The ALFA system consists of four stations with detectors in an upper and lower Roman Pots (RP). In total, eight ALFA detectors are used in the system. Two stations are placed on each side of the ATLAS interaction point at a distance about $240 \mathrm{~m}$. The distance between two stations was approximately 4 m during Run 1 .

A single ALFA detector consists of Main Detector (MD), Overlap Detector (OD) and trigger detectors, see Fig. 1 left panel. MD is made of 20 staggered layers of square-shaped $0.5 \mathrm{~mm}$ thick scintillating fibres, 64 fibres in each layer. Fibres are inclined by $+45^{\circ}$ (10 layers) and $-45^{\circ}(10$ layers) with respect to vertical direction. Resolution of MD is of about $30 \mu \mathrm{m}$ thanks to staggering of the layers. OD consists of fibres which are arranged horizontally and measure the vertical coordinate only with a precision of $100 \mu \mathrm{m}$. MD and OD are readout by Multi-Anode PhotoMultiplier Tubes. Both MD and OD are equipped with scintillator tiles for triggering. The tiles are made of $3 \mathrm{~mm}$ thick plastic scintillator, which have the shapes of the corresponding sensitive areas of MD and OD.

The ALFA detectors are installed in a secondary vacuum inside the RP for safe separation from the ultra-high vacuum of LHC. The RP, see Fig.1 right panel, consists of a cylindrical pot, a cavern for the detector and two flanges. The cylindrical flange is connected to the bellow and allows the movement of RP. The squared flange is used to fix the detector base plate. The RP is made of stainless steel. The side walls are $500 \mu \mathrm{m}$, bottom window is $200 \mu \mathrm{m}$ thick. The RP can approach vertically the LHC beam to milimeter distance. In Run 1, there was a semi-circular ferrite tile attached to the bottom of the cylindrical part of the RP.
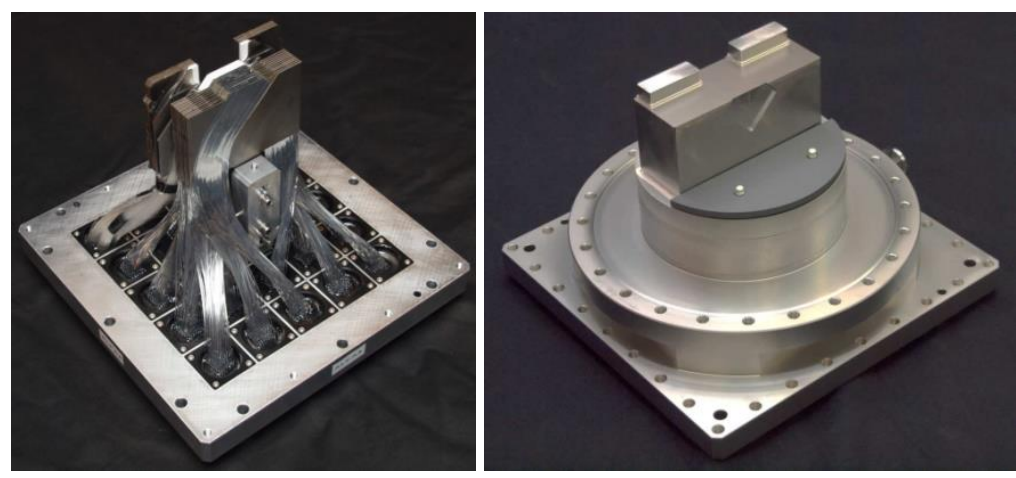

Figure1. The ALFA detector is made of scintillating fibres which are read out with MAPMTs (left panel). Roman Pot (right panel) separates the secondary vacuum volume where the detector is housed from the ultra-high vacuum of the LHC beam-pipe interior. 


\section{Detector heating in Run 1}

The ferrite tile attached to the bottom of the RP aims to absorb the electromagnetic power of the RF wake field caused by repetitive passing of the proton bunches by the RP. The field resonates in the cavity where the RP are installed. The resonances are the origin of the RF losses, the power deposition to the RP and heating up the detector.

During LHC Run 1 fill the estimated power deposit was $10 \mathrm{~W}$ and the detectors were heated up to $45^{\circ} \mathrm{C}$. The titanium substrates and the fibres glued on them have different thermal expansion coefficients and the resulting stress can destroy the assembly. Extrapolations to Run 2 indicated a power deposit of up to $80 \mathrm{~W}$ in case of extreme beam conditions.

\section{ALFA detector system improvements for Run 2}

The main effort between Run 1 and Run 2 was dedicated to the heat protection, other modifications were a displacement of the two outer stations and the improvement of trigger capabilities.

\subsection{RP-filler}

To minimize the RF impact on the temperature four measures were taken. The most important was to reduce the cavity volume, and thereby the RF power deposit, by extending the RP by a so-called RP-filler. In addition, the ferrites were mounted at positions where they absorb the wake fields of the beams in more efficient way, a heat distribution system made of thin copper plates was installed inside the RP and the air cooling was intensified by additional fans and a better streaming to the RP flanges. The three first modifications are shown in Fig. 2.

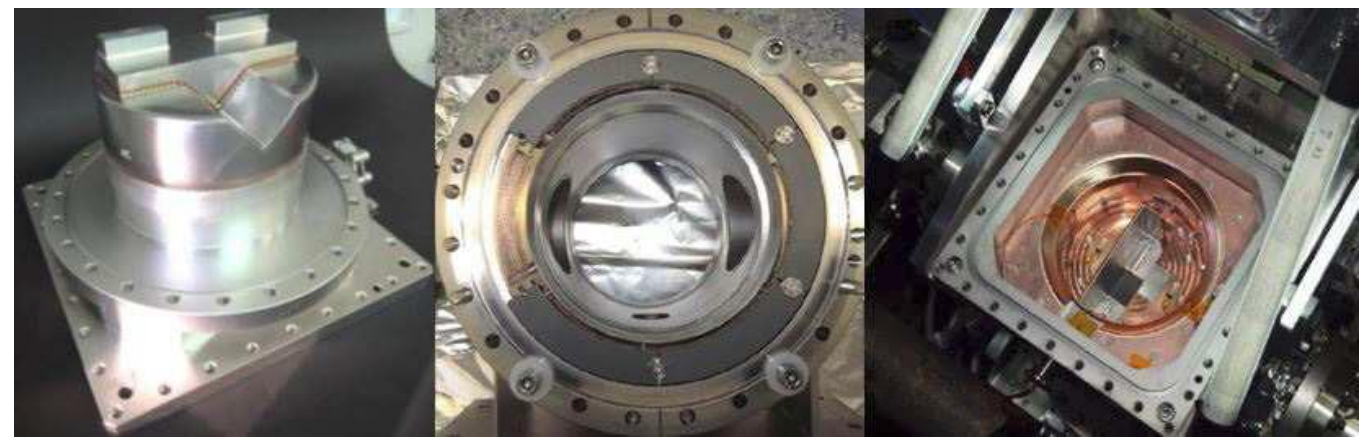

Figure 2. Three modifications to improve the heat protection. From left to right: the RP-filler attached to the bare RP, the ring of ferrites distributed on the RP station flange and the copper heat distribution system with attached temperature probes.

The RP-filler is made of titanium and connected by conductive copper-beryllium springs to the original RP. Thanks to the RP-filler all resonances between $0.6 \mathrm{GHz}$ and $1.8 \mathrm{GHz}$ are strongly suppressed. In particular, those below $1 \mathrm{GHz}$, which have the strongest coupling to the beam spectrum and are most dangerous for heating, disappeared completely.

The semi-circular ferrite tile at the RP bottom has been replaced by a ring of six individual pieces located on the flange. At the new positions the heat is transmitted to the flanges where the air stream for cooling is circulating.

After extensive lab tests, the stations were reinstalled in the LHC tunnel. The temperature increase in a high intensity fill is below $5^{\circ} \mathrm{C}$ (see Fig. 3), far from the destructive range. 


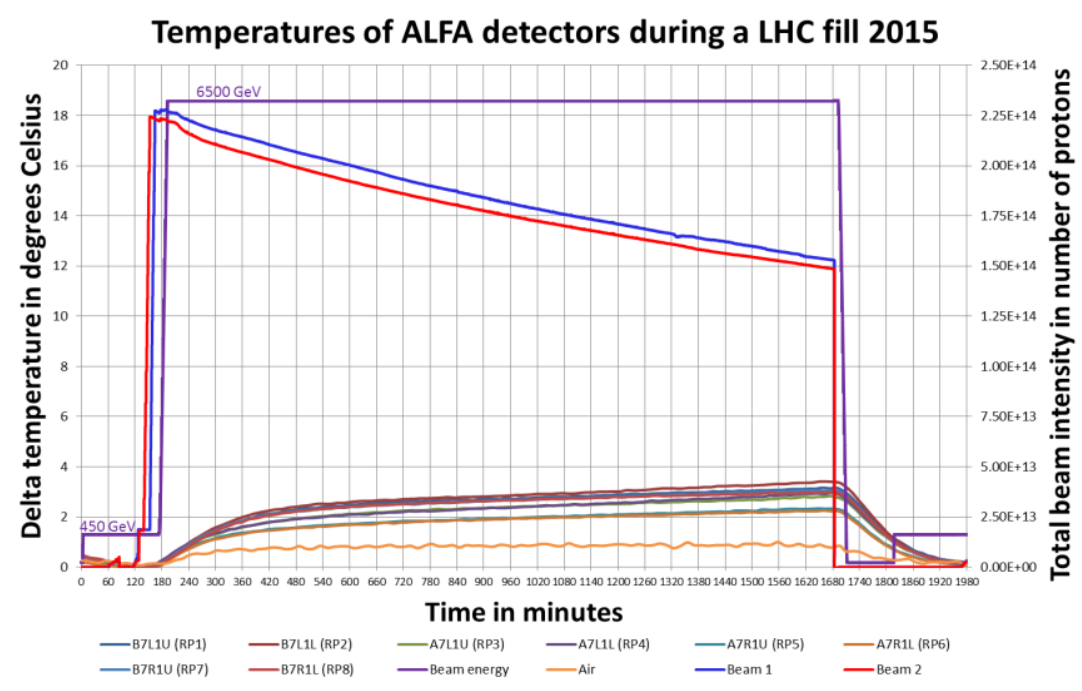

Figure 3. Record of temperature changes in high intensity fill proves efficiency of the measures for minimizing the detector heating. The temperature increase does not exceed $5^{\circ} \mathrm{C}$.

\subsection{Enlarged distance between inner and outer stations}

The outer stations were displaced downstream from their original positions. In this way the distance between inner and outer station was enlarged from $4 \mathrm{~m}$ to $8 \mathrm{~m}$. The angular resolution was improved by a factor two which results in a higher precision of the reconstructed proton momentum transfer.

\subsection{Trigger upgrade}

Dead time was reduced from $550 \mathrm{~ns}$ to $88 \mathrm{~ns}$ thanks to upgrades in the firmware of the MAROC chip of the front end electronics. This allows triggering and data taking with the LHC bunch spacing down to $100 \mathrm{~ns}$.

Upgrade of the ATLAS Central Trigger Processor (CTP) brought a latency penalty $75 \mathrm{~ns}$ what increased the ALFA latency beyond the limits of the first level trigger (L1) budget. For compensation a new back-end trigger board was produced, allowing a by-pass of the standard trigger input stages and injection of signals directly to CTP core. In addition, it replaces the NIM electronics used in the past for separation of MD and OD trigger signals and allows the monitoring of rates per bunch crossing.

\section{References}

[1] The ATLAS Collaboration, The ATLAS Experiment at the CERN Large Hadron Collider, JINST 3 (2008) S08003.

[2] The ATLAS Collaboration, ATLAS Forward Detectors for Measurement of Elastic Scattering and Luminosity, ATLAS-TDR-18, CERN-LHCC-2008-004 (2008), http://cds.cern.ch/record/1095847.

[3] The ATLAS Collaboration, Measurement of the total cross section from elastic scattering in $p p$ collisions at $\sqrt{s}=7$ TeV with ATLAS detector, Nucl. Phys. B 889 (2014) 486-548.

[4] The ATLAS Collaboration, Measurement of the total cross section from elastic scattering in $p p$ collisions at $\sqrt{s}=8 \mathrm{TeV}$ with ATLAS detector, Phys. Lett. B 761 (2016) 158-178. 